Supporting Information

Fair Investigation of Cross-link Density Effects

on the Bond-exchange Properties for Trans-esterification-based Vitrimers

with Identical Concentration of Reactive Groups

Mikihiro Hayashi*, Ryoto Yano

Department of Life Science and Applied Chemistry, Graduated School of Engineering, Nagoya Institute of Technology, Gokiso-cho, Showa-ku Nagoya 466-8555, Japan

1. Synthesis of PE-COOH

2. TGA thermograms

3. WAXD profiles

4. Reproducibility of stress-strain data

5. Quantitative analysis of the modulus

6. Dilatometry test

7. Reproducibility of stress-relaxation data

8. Stress-relaxation at $150{ }^{\circ} \mathrm{C}$ 


\section{Synthesis of PE-COOH}

Polyesters with multiple $\mathrm{COOH}$ side groups (PE-COOH) were synthesized according to our previous report (see Scheme S1). First, melt polycondensation was conducted with using 1,5-pentanediol (PD), thiomalic acid (TMA), adipic acid (AdA) with a small amount of $\mathrm{Sc}(\mathrm{OTf})_{3}$ catalyst. In the polycondensation, the stoichiometric ratio between $\mathrm{COOH}$ and $\mathrm{OH}$ was kept to be unity while the mole ratio of TMA to AdA was set to be PD/AdA/TMA = $1 / 0.7 / 0.3$. The polycondensation was carried out at $80{ }^{\circ} \mathrm{C}$ for approximately 16 hours with vacuum stripping. Purification was conducted by precipitating its THF solution into methanol repeatedly. We then reacted acrylic acid with $\mathrm{SH}$ group of the above polyester via Michael addition reaction. The polyesters with $\mathrm{SH}$ groups were dissolved in DMF, and then acrylic acid ( 3 eq. to SH groups in the polyester) and triethylamine ( 0.33 eq. to $\mathrm{SH}$ groups in the polyester) were added. The reaction was kept at room temperature overnight. Unreacted acrylic acid monomers were removed by re-precipitation of the THF solution into methanol repeatedly.

Scheme S1. Synthesis of PE-COOH

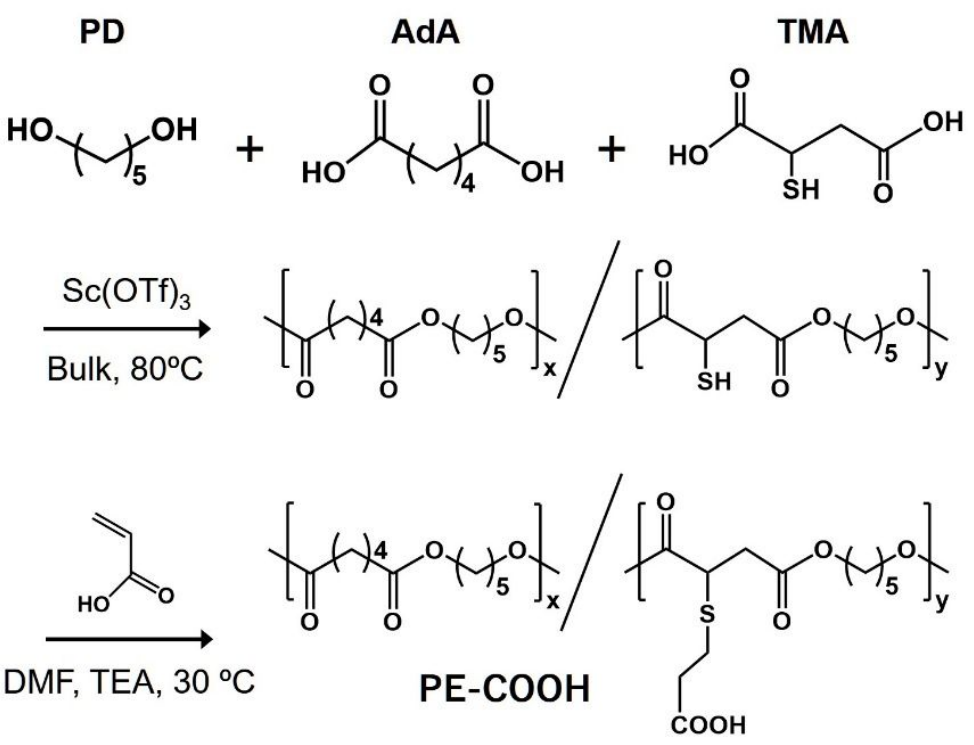

${ }^{1} \mathrm{H}-\mathrm{NMR}$ measurements were taken to determine the unit ratio of PD/AdA/TMA in the polyesters synthesized by polycondensation reaction. The spectra for the polyester with SH side groups is provided in Figure S2 (black). Integral values on the signals are presented in Table S1. Similarly, the polyester with $\mathrm{COOH}$ side groups $(\mathrm{PE}-\mathrm{COOH})$ synthesized via Michael addition is provided in Figure S2 (red). Hx with alphabet (x) in the spectra corresponds to the protons with the same alphabet in the chemical structures shown in Figure S1. 

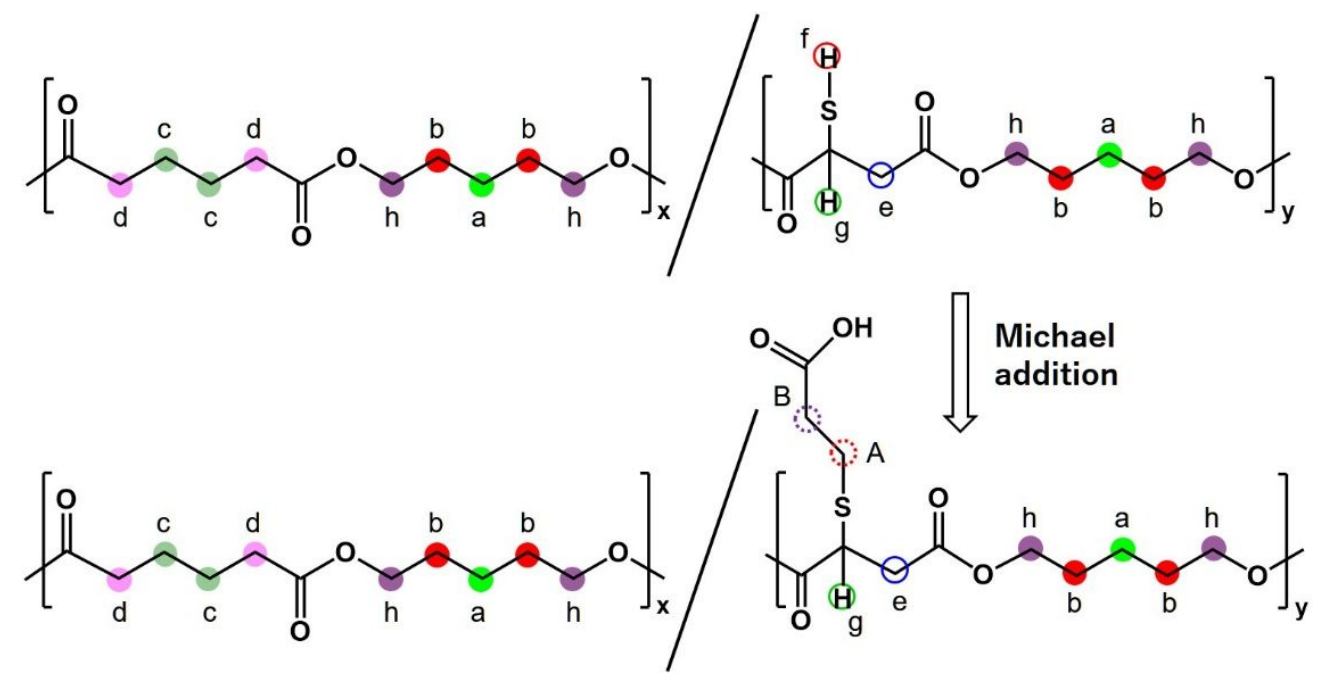

Figure S1. Assignment for proton signals for ${ }^{1} \mathrm{H}-\mathrm{NMR}$ spectra in Figure S2.

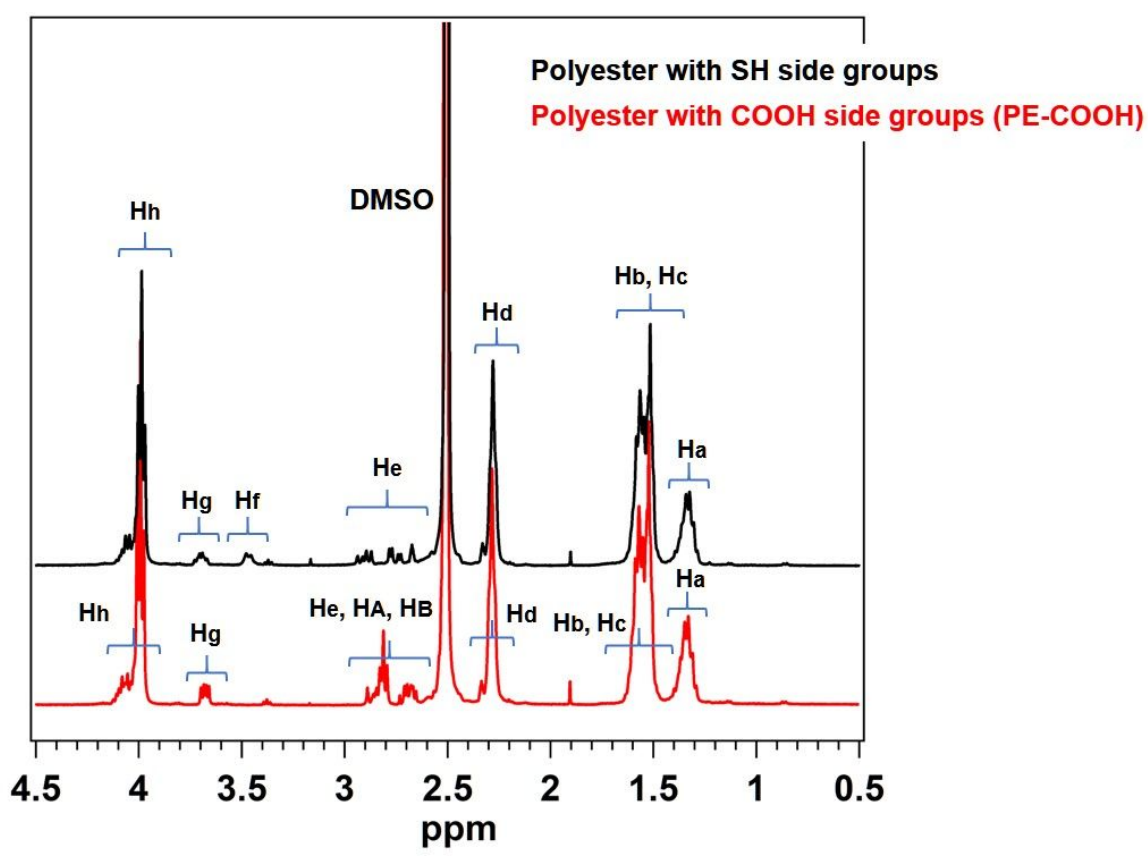

Figure S2. ${ }^{1} \mathrm{H}-\mathrm{NMR}$ spectra for polyester with $\mathrm{SH}$ side groups (black) and $\mathrm{COOH}$ side groups (red). 
Table S1. Attribution of signals and integral values for polyester with $\mathrm{SH}$ groups.

\begin{tabular}{|l|l|c|c|}
\hline & \multicolumn{1}{|c|}{$\sigma(\mathrm{ppm})$} & integral found & expected \\
\hline $\mathrm{H}_{\mathrm{a}}$ & $1.3-1.4$ & 1.00 & 1.00 \\
\hline $\mathrm{H}_{\mathrm{b}}, \mathrm{H}_{\mathrm{c}}$ & $1.5-1.6$ & 3.18 & 3.30 \\
\hline $\mathrm{H}_{\mathrm{d}}$ & $\sim 2.4$ & & \\
\hline $\mathrm{H}_{\mathrm{e}}$ & $2.7-3.0$ & 1.33 & 1.40 \\
\hline $\mathrm{H}_{\mathrm{f}}$ & $\sim 3.45$ & 0.23 & 0.29 \\
\hline $\mathrm{H}_{\mathrm{g}}$ & $\sim 3.7$ & 0.11 & 0.11 \\
\hline $\mathrm{H}_{\mathrm{h}}$ & $3.9-4.1$ & 0.12 & 0.11 \\
\hline
\end{tabular}

From the integral ratios, we confirmed that the unit ratios of PD/AdA/TMA are approximately the same as the feed mole ratios (see Table S1). We estimated the number of SH groups $\left(N_{\mathrm{SH}}\right)$, which corresponds to $N_{\mathrm{COOH}}$ in $\mathrm{PE}-\mathrm{COOH}$, from the unit ratios and $M_{\mathrm{n}}$ determined by SEC measurements. In the polyester, there are two kinds of unit; one is composed of TMA and PD (Unit-TMA), and the other is composed of AdA and PD (Unit-AdA). By using $M_{\mathrm{n}}$ from SEC and each unit mass (218.3 for Unit-TMA and 214.3 for Unit-AdA), we obtain the equation $\mathrm{S} 1$, $M_{\mathrm{n}}=218.3 \times n_{\mathrm{TMA}}+214.3 \times n_{\mathrm{AdA}} \quad(\mathrm{S} 1)$,

where $n_{\mathrm{TMA}}$ and $n_{\mathrm{AdA}}$ represents the number of unit per chain for Unit-TMA and Unit-AdA, respectively. In addition, the ratio between $n_{\mathrm{TMA}}$ and $n_{\mathrm{AdA}}$ can be estimated from the ${ }^{1} \mathrm{H}-\mathrm{NMR}$. This leads to

$n_{\mathrm{TMA}}: n_{\mathrm{AdA}}=x: y$

, where $x$ and $y$ is the values of the ratio. Finally, the simultaneous equation of S1 and S2 is solved to provide the values of $n_{\mathrm{TMA}}$ and $n_{\mathrm{AdA}}$, leading $N_{\mathrm{SH}}\left(=N_{\mathrm{COOH}}=n_{\mathrm{TMA}}\right)$. 


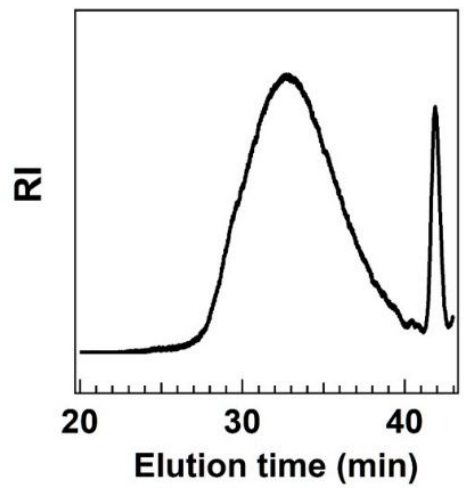

Figure S3. SEC curve for the precursor polyester bearing thiol groups. 


\section{TGA thermograms}

Figure S4 represents the TGA thermograms for PE-COOH and CL-PE- $X$ (see the measurement condition in the main text).
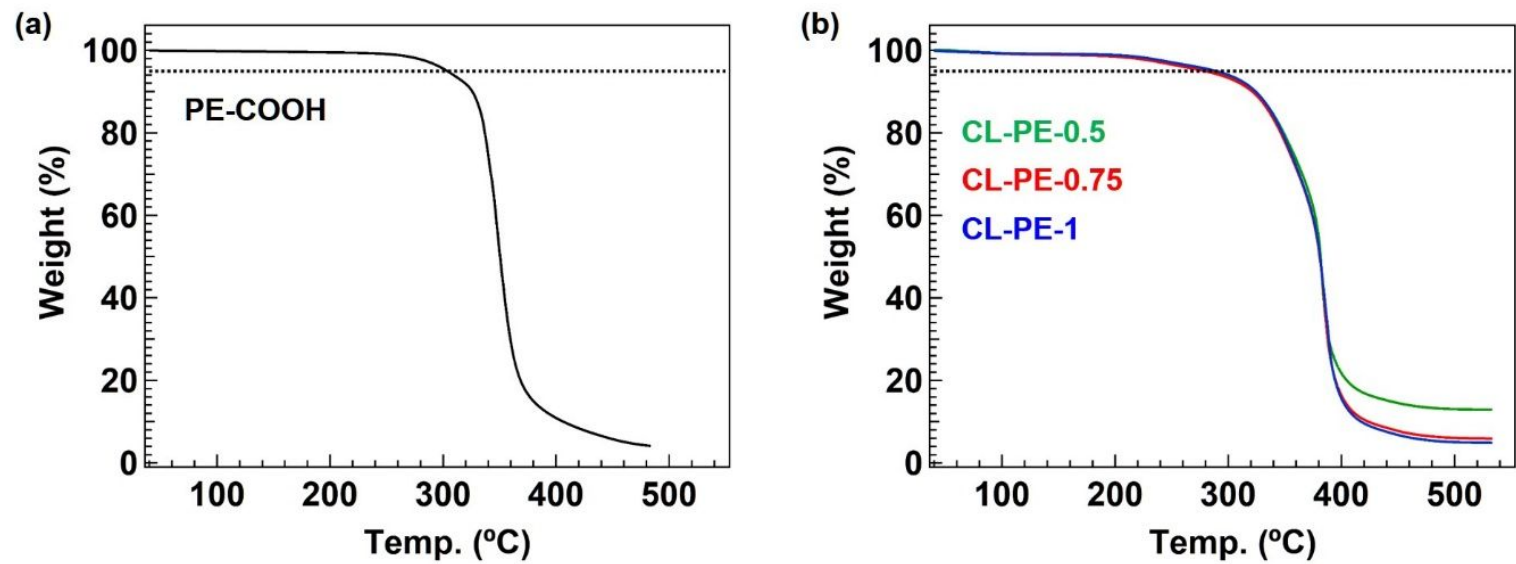

Figure S4. TGA thermograms for (a) PE-COOH and (b) CL-PE- $X$. 


\section{WAXD profiles}

Figure S5a summarizes WAXD profiles for CL-PE- $X$ (see the measurement condition in the main text). For comparison, we prepared blend of $\mathrm{PE}-\mathrm{COOH}$ and $\mathrm{Zn}(\mathrm{OAc})_{2}$ by direct mixing without using solvents, and the profile is shown in Figure S5b. In each profile for CLPE- $X$, only broad amorphous halo was observed while there were multiple sharp peaks observed for the direct mixing blend. According to the profile taken for $\mathrm{Zn}(\mathrm{OAc})_{2}$ powder sample capped with kapton tapes, the sharp peaks observed in the direct mixing blend originated from the aggregated clusters of crystallized $\mathrm{Zn}(\mathrm{OAc})_{2}$. These data revealed that $\mathrm{Zn}(\mathrm{OAc})_{2}$ was well dispersed without forming aggregated clusters for CL-PE- $X$ that was prepared by solvent casting method.
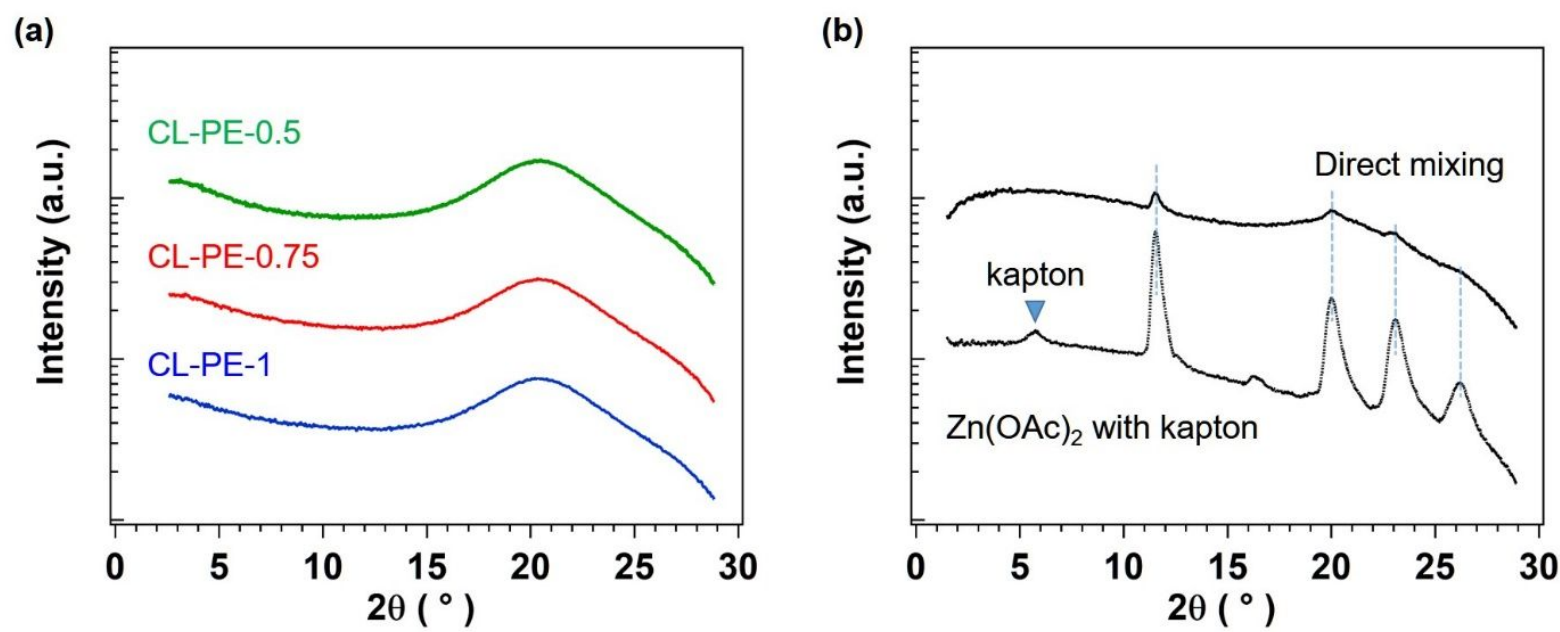

Figure S5. WAXD profiles for (a) CL-PE- $X$ and (b) the blend prepared by direct mixing. In (b), the profile taken for $\mathrm{Zn}(\mathrm{OAc})_{2}$ powder sample capped with kapton tapes is shown for comparison. 


\section{Reproducibility of stress-strain data}

Figure S6 shows the stress-strain data measured for three different samples of CL-PE-1 to check the reproducibility of the data. Although some deviation were observed between data at the large deformation region, the modulus estimated at the low deformation region was similar.

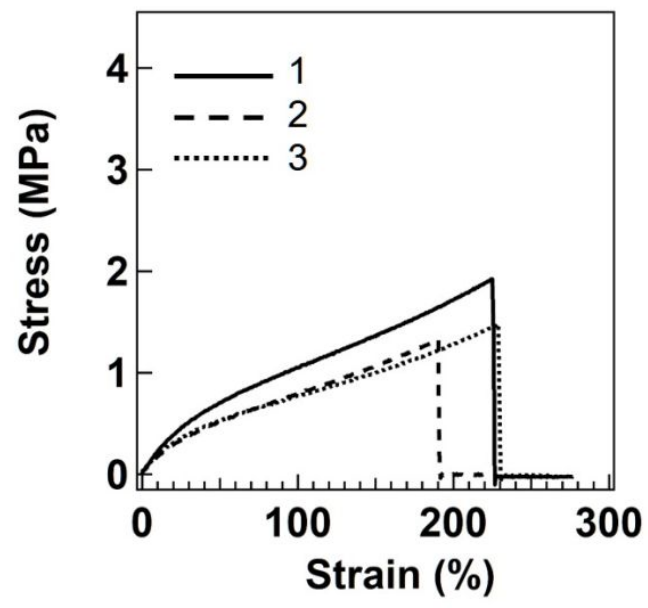

Figure S6. Stress-strain curves for CL-PE-1 measured for three different samples. 


\section{Quantitative analysis of the modulus}

Firstly, the modulus in the affine network model $\left(E_{\text {aff }}\right)$ is expressed as equation $(\mathrm{S} 1)$ with using molecular weight between cross-links $\left(M_{\mathrm{X}}\right),{ }^{1}$ where the Poisson ratio $v=0.5$ is used in the relationship of $E=2 G(1+v)$.

$$
E_{a f f}=\frac{\rho}{M_{x}}(3 R T)
$$

In equation (S1), $R, T$ and $\rho$ represents the gas constant, the measurement temperature, and the density, respectively. In addition, there is another modified model, known as the phantom network model, which considers effect of the fluctuation of cross-link points during elongation. In the phantom network model, the modulus $\left(E_{\mathrm{ph}}\right)$ is expressed as equation $(\mathrm{S} 2){ }^{1}$

$$
E_{p h}=\frac{\rho}{M_{x}}(3 R T)\left(1-\frac{2}{f}\right)
$$

, where $f$ is the number of branch at a cross-link point ( $f=3$ in the present design). In this molecular design, $M_{\mathrm{X}}$ can correspond to $M_{\mathrm{COOH}},\left(=\mathrm{COOH}\right.$ group equivalent weight $=M_{\mathrm{n}} /$ $\left.N_{\mathrm{COOH}}=740\right)$ if the ideal network is formed where all $\mathrm{COOH}$ groups in $\mathrm{PE}-\mathrm{COOH}$ are intermolecularly reacted with the diepoxy cross-linkers. Therefore, we estimated the ideal elastic modulus based on the phantom network model $\left(E_{\text {ideal,ph }}\right)$ by substituting $M_{\mathrm{X}}=M_{\mathrm{COOH}}$ for equation (S2), where the contribution from the inorganic catalyst was not taken into account due to the very low weight concentration. For the present sample cross-linked with finite values of $f_{\text {di-epoxy }}$, the ideal modulus $E_{\text {ideal,ph }}$ is approximately expreseed by using $f_{\text {di-epoxy, }}$,

$E_{\text {ideal }, p h}=\frac{\rho}{M_{x}}(3 R T)\left(1-\frac{2}{f}\right) \times f_{\text {di-epoxy }}$

Figure S7 compared the experimental $E_{\mathrm{Y}}$ (red plots) with $E_{\text {ideal,ph }}$ (block broken line) as a function of $f_{\text {di-epoxy. }}$. The experimental $E_{\mathrm{Y}}$ was much smaller than $E_{\text {ideal,ph. This suggests there are }}$ some defects of network, such as dangling chain, loop formation, and unwilling intramolecular epoxy-COOH reaction.

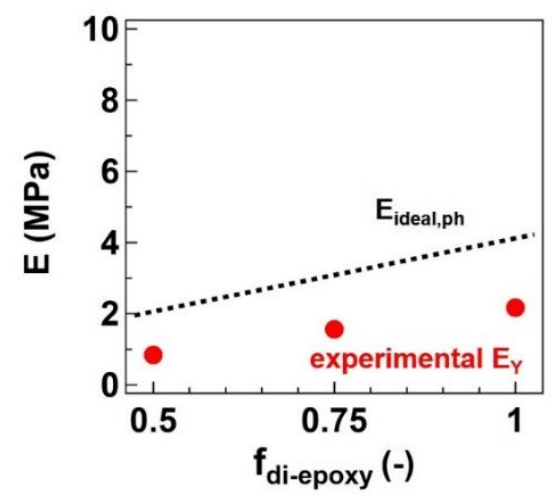

Figure S7. Comparison of the experimental $E_{\mathrm{Y}}$ (red plots) with $E_{\text {ideal,ph }}$ (block broken line).

1) M. Rubinstein and R. Colby, Polymer Physics, Oxford University Press, New York, 2003. 


\section{Dilatometry test}

Dilatometry was performed to estimate the temperature for activating bond-exchange (see the measurement condition in the main text). It has been conventionally known that the sample length is suddenly deviated from the constant sample length change (i.e., sample thermal expansion) when the bond-exchange begins to be activated. In the present samples CL-PE- $X$, the deviation (i.e., softening) was observed at approximately $150{ }^{\circ} \mathrm{C}$ (Figure S8a). Therefore, the bond-exchange was judged to be sufficuently activated at temperatures higher than $150{ }^{\circ} \mathrm{C}$. On the other hand, the control sample, that is, the cross-linked sample with no $\mathrm{Zn}(\mathrm{OAc})_{2}$ catalysts, did not show the softening (Figure S8b), meaning the observed softening for the present samples were indeed due to the catalytic activation of trans-esterification.

(a)

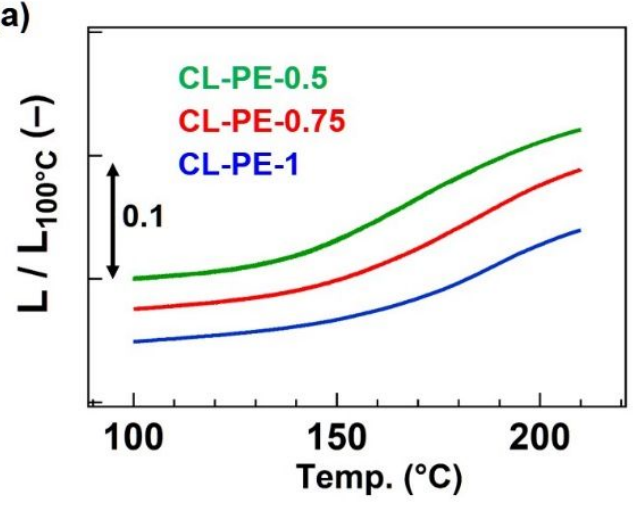

(b)

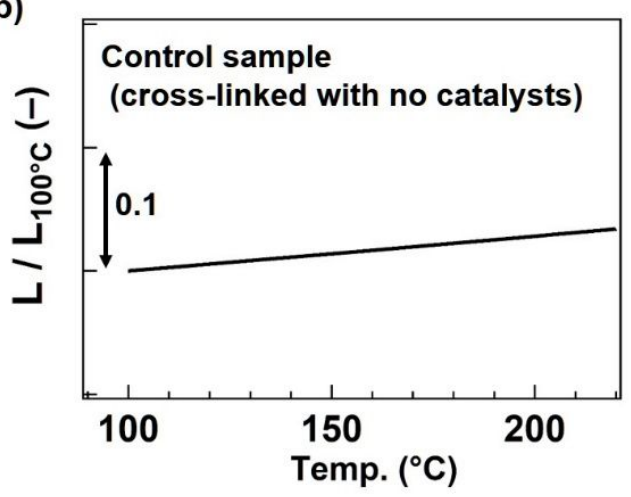

Figure S8. Changing behavior of the sample length during the temperature increase under a constant tension $(30 \mathrm{mN}$ ) for (a) CL-PE- $X$ and (b) the control sample cross-linked without no $\mathrm{Zn}(\mathrm{OAc})_{2}$ catalysts. The y-axis indicates the sample length $(L)$ from measurements normalized by the length at $100{ }^{\circ} \mathrm{C}\left(L_{100^{\circ} \mathrm{C}}\right)$. In (a), the curves were shifted vertically for a clearer comparison. 


\section{Reproducibility of stress-relaxation data}

Figure S9 shows the reproducibility of stress-relaxatoin data. Due to the limitation of sample amounts, the measurements were performed for CL-PE- 1 at $170{ }^{\circ} \mathrm{C}$ and for CL-PE-0.5 at $160{ }^{\circ} \mathrm{C}$.

(a) CL-PE-1, $170^{\circ} \mathrm{C}$

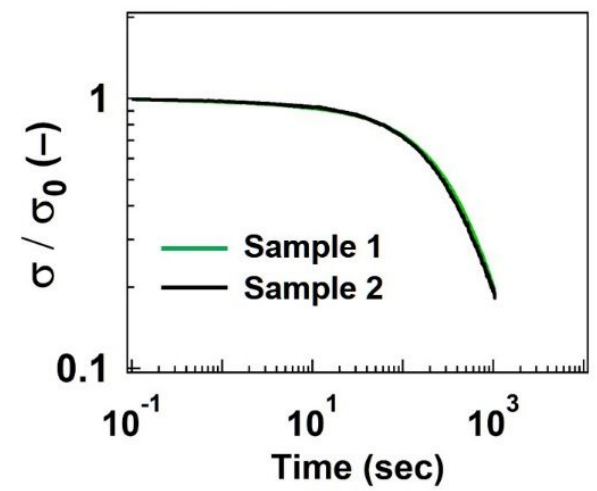

(b) CL-PE-0.5, $160^{\circ} \mathrm{C}$

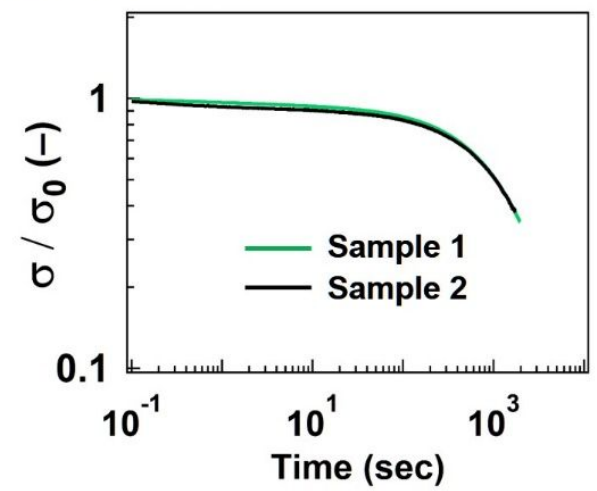

Figure S9. Reproducibility of stress-relaxatoin data measured for (a) CL-PE-1 at $170{ }^{\circ} \mathrm{C}$ and for (b) CL-PE-0.5 at $160{ }^{\circ} \mathrm{C}$. 


\section{Stress-relaxation at $150{ }^{\circ} \mathrm{C}$}

Figure $\mathrm{S} 10$ represents the stress-relaxation data at $150{ }^{\circ} \mathrm{C}$. The relaxation time $\langle\tau\rangle$ is also summarized in Table S2.

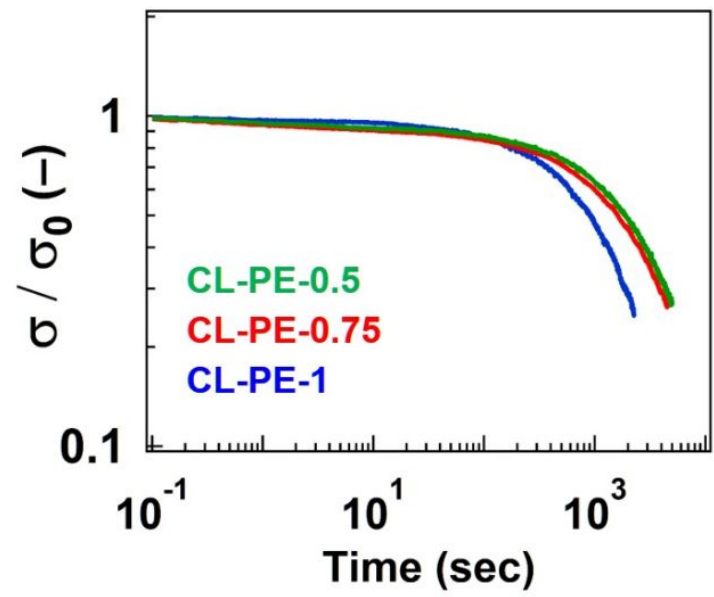

Figure S10. Stress-relaxation data measured at $150{ }^{\circ} \mathrm{C}$.

Table S2. The relaxation time $<\tau>$ at $150^{\circ} \mathrm{C}$

\begin{tabular}{|c|c|c|c|}
\hline & CL-PE-1 & CL-PE-0.75 & CL-PE-0.5 \\
\hline$<\tau>$ a $/$ sec & 1875.6 & 4948.7 & 5202.4 \\
\hline
\end{tabular}

a) relaxation time estimated by fitting analysis. 